\title{
Intergenerational transmission of pathogenic heteroplasmic mitochondrial DNA
}

\author{
Lee-Jun C. Wong, PhD' ${ }^{1}$, Hansie Wong, BA ${ }^{1}$, and Aiyi Liu, $P h D^{2}$
}

\begin{abstract}
Purpose: To study the pattern of intergenerational transmission of pathogenic mitochondrial DNA with heteroplasmic A3243G, G8363A, A8344G, T8993G, and T8993C mutations. Methods: The mutant load in the carrier mother and her offspring was measured in a total of 79 transmissions. Statistical analysis was performed to determine whether the intergenerational change in heteroplasmic mutant mtDNA is significant. Results: Our results demonstrate that A3243G and T8993G mutant mtDNAs are significantly increased in blood, hair follicles, and buccal mucosal cells, during intergenerational transmission, whereas the intergenerational increase in T8993C and A8363G mutant mtDNA is not significant. Unlike previous reports, in one large family with A8344G mutation, the mutant load was slightly increased, instead of decreased, during transmission. There is no significant difference in the intergeneration transmission of mutant mtDNA to male or female offspring. Conclusion: Intergenerational transmission of heteroplasmic A3243G and T8993G mtDNA results in significant increase of mutant mtDNA in the offspring. Transmission of T8993C, G8363A, and A8344G does not result in significant intergenerational change in mutant load. Tissue specificity and genetic background may play important roles in the transmission of pathogenic heteroplasmic mtDNA. Genet Med 2002:4(2):78-83.
\end{abstract}

Key Words: heteroplasmic mtDNA, mtDNA mutation, transmission of mtDNA, mitochondrial DNA, mitochondrial genetics

In addition to the nuclear genome, mammalian cells contain multiple copies of circular, double-stranded DNA in the individual mitochondrion, a cytoplasmic organelle whose major function is to produce ATP through oxidative phosphorylation. ${ }^{1}$ There are approximately 85 protein polypeptides that make up the five complexes of the oxidative respiratory chain. Only 13 are encoded by the mitochondrial genome. The remaining polypeptides are encoded by nuclear genes (nDNA). Therefore, mitochondrial disorders can be caused by mutations in either the nuclear or the mitochondrial genome. The mitochondrial DNA (mtDNA) -encoded polypeptides were synthesized on mitochondrial ribosomes using mitochondrial-specific transfer RNAs. The 16.6-kb mitochondrial genome encodes 2 rRNAs, 22 tRNAs, 13 mRNAs, and approximately 1 $\mathrm{kb}$ of noncoding sequence (the D loop region). Each cell contains hundreds to thousands of mitochondria. Unlike the Mendelian inheritance of the nuclear genes, the segregation of mitochondrial genomes is random and it is exclusively maternally inherited. Because mtDNA is not coated by protective histones and is constantly exposed to the high concentration of reactive oxygen species due to the close proximity to the inner

From the ${ }^{1}$ Institute for Molecular and Human Genetics, ${ }^{2}$ Biostatistics Unit, Lombardi Cancer Center, Georgetown University Medical Center, Washington, DC.

Lee-Jun C. Wong, PhD, Institute for Molecular and Human Genetics, Georgetown University Medical Center, M4000, 3800 Reservoir Road, NW, Washington, DC 20007.

Received: November 9, 2001.

Accepted: December 17, 2001. mitochondrial membrane where electron transport takes place, the mtDNA has a high mutation rate. Because of the multiplicity of mitochondria per cell, the mutant mtDNA may coexist with the wild-type mtDNA, a phenomenon known as heteroplasmy. There are numerous mtDNA single nucleotide polymorphisms that are usually homoplasmic and benign. In humans, the heteroplasmic mutant mtDNA are usually associated with disease. ${ }^{1-3}$ The most common disease-causing mtDNA mutations are A3243G in tRNA ${ }^{\text {Leu(UUR) }}$ for MELAS (mitochondrial myopathy, encephalopathy, lactic acidosis, and stroke-like episodes), A8344G in tRNA ${ }^{\text {Lys }}$ for MERRF (myoclonic epilepsy and ragged-red fibers), and T8993G/C (ATPase subunit 6) for NARP (neuropathy, ataxia, and retinitis pigmentosa, and Leigh's syndrome). More recently, the G8363AtRNA ${ }^{\text {Lys }}$ mutation causing cardiomyopathy, peripheral neuropathy, and hearing loss, was found in two large families. ${ }^{4}$ High levels of mutant mtDNA are often correlated with devastating neuromuscular disorders. ${ }^{5,6}$

An asymptomatic carrier woman can transmit a variable amount of mutant mtDNA to her offspring with a broad range of clinical features from severely to mildly or not affected. With an increasing number of patients being diagnosed with mtDNA disorders, there is a demand to offer the at-risk families accurate counseling on disease prognosis, recurrence risk, usefulness of prenatal diagnosis, and possible prevention. However, these processes are greatly hindered by the inability of predicting the trend of transmission of mutant mtDNA and the severity and age of onset of the disease, due to the unique features of heteroplasmic 
multiple copies of mtDNA and tissue threshold effect. Additionally, there is tissue-specific selection for or against the mutant mtDNA. Therefore, the level of mutant mtDNA varies among tissues and may also change over time..$^{7-10}$

The intergenerational change in the level of heteroplasmic mutant mtDNA and the variation in multisystemic phenotypic expression are determined by two major processes: the genetic bottleneck during the formation of mature oocytes and the differential replication and segregation of mtDNA during histogenesis and organ maturation. ${ }^{11,12}$ The genetic "bottleneck" may cause a small proportion of the mitochondrial genome to repopulate the offspring. Thus, a germ-line bottleneck may lead to a rapid genetic drift with either the loss of the mutation completely or offspring with homoplasmic mutant mtDNA. ${ }^{13,14}$ Because the size of the bottleneck is undefined, it is difficult to predict the level of mutant mtDNA in the offspring or the clinical outcome of patients with a particular mutation. ${ }^{15}$ In somatic cells, there is a tissue-specific replicative selection such that mutant mtDNA could either increase or decrease during the postmitotic growth. ${ }^{7-10}$ To understand the inheritance of the heteroplasmic mtDNA mutations, the relationship of disease expression in the offspring and the mutant load of the carrier mother with A3243G and $\mathrm{A} 8344 \mathrm{G}$ mutation has been studied. ${ }^{16}$ It was found that the frequency of clinically affected offspring born to women who harbor the $\mathrm{A} 3243 \mathrm{G}$ or the $\mathrm{A} 8344 \mathrm{G}$ point mutation was related to the level of mutant mtDNA in the mothers' blood. ${ }^{16}$ However, at any one level of maternal mutant load, there was a greater frequency of affected offspring for the A3243G mutation than for the A8344G mutation. Studies of the importance of longitudinal and tissuespecific effects on differential selection of mutant mtDNA have shown negative selection of pathogenic A3243G and G3460A mutations in blood cells. ${ }^{8,17}$ Yet, Yoneda et al. found replicative advantage of A3243G mutant mtDNA in myoblast cultures. ${ }^{10}$ In addition, the mtDNA mutations at nucleotide position (np) 8993 showed lack of tissue-specific and age-related variations. ${ }^{18}$ Recent investigation of the inheritance of the six most common pathogenic heteroplasmic mutations revealed a preferential transmission of A3243G, T8993G, and G11778A mutant mtDNAs. ${ }^{19}$ On the contrary, the transmission of the $\mathrm{A} 8344 \mathrm{G}$ mutation was negatively selected. ${ }^{19}$ These studies measured the mutant loads in blood only. Additional data on studies of more unrelated families with large number of transmissions in blood and other tissues would help to support their observations and to resolve the discrepancies. In this report, we studied a total of 79 intergenerational transmissions of five different pathogenic mtDNA mutations, including the transmission of G8363A mutation that was discovered recently. ${ }^{4}$ We also examined the pattern of intergenerational transmission of mutant mtDNA in hair follicles and buccal cells.

\section{MATERIALS AND METHODS}

\section{Patients}

Patients suspected of mitochondrial disorders were referred to our laboratory for comprehensive mutational analysis of mtDNA that included 11 common point mutations and
Southern analysis of mtDNA deletions and rearrangement. ${ }^{20-22}$ Only the transmissions of heteroplasmic pathogenic mutations from carrier mother to her offspring were included in this study. Patients with sporadic mutations without a carrier mother were not included. The patients are of various ethnic background, including Asians, African Americans, Caucasians, and Hispanics. Overall, 109 patients with a total of 79 transmissions were analyzed.

\section{Mutation detection and quantification}

Multiplex PCR followed by allele-specific oligonucleotide (ASO) dot blot analysis was used to detect point mutations in mtDNA. ${ }^{20,21}$ This method is sensitive enough to detect a mutant load as low as $2 \% .{ }^{20}$ Once a mutation is detected by ASO, the proportion of mutant mtDNA is quantified by restriction fragment length polymorphism (RFLP) analysis according to published procedures. ${ }^{4,23-25}$ The restriction enzymes used for A3243G, A8344G, G8363A, T8993G, and T8993C were HaeIII, BglI, HphI, AvaI, and MspI, respectively. ${ }^{4,23-25}$ Briefly, the PCR products were labeled with $\left[\alpha-{ }^{32} \mathrm{P}\right] \mathrm{dCTP}$ in the last cycle PCR followed by restriction enzyme digestion. ${ }^{26}$ The detailed conditions have been described in the literature. ${ }^{4,23-26}$ Most of the amplified fragments contain at least one additional restriction site as internal control to ensure that enzyme digestion is complete. The amount of radioactivity in the mutant and wild-type bands was measured using Betascope 603.23 At least two independent measurements of two DNA extractions from each sample were performed. Mutant loads in muscle, blood lymphocytes, and other noninvasive tissues such as hair follicle and buccal cells were also measured when available. Because the muscle specimen from the asymptomatic carrier mother was usually unavailable, the intergenerational transmission was studied in the noninvasive tissues only.

\section{Statistical analysis}

The mutant load in each specimen was the mean of $2-10$ independent measurements done at different times. The coefficient of variation $(\mathrm{CV})$ varies from sample to sample, depending on the mutant loads. They were between 0 and 0.07 , 0.01 to 0.38 , and 0.19 to 0.53 with an average $\mathrm{CV}$ of $0.03,0.13$, and 0.36 , for mutant loads above $70 \%, 10$ to $70 \%$, and below $10 \%$, respectively. The mean mutant load is used for statistical analysis. The descriptive statistics (mean and standard deviation) was derived for intergenerational changes of each mutation. Because the sample size was relatively small, the nonparametric Wilcoxon rank-sum test was used to analyze the data. ${ }^{27}$

\section{RESULTS}

We studied the transmission of five heteroplasmic pathogenic mtDNA mutations: A3243G (10 families with a total of 30 transmissions); A8344G (1 family with 19 transmissions); G8363A (1 family with 11 transmissions); T8993G (4 families with 6 transmissions); and T8993C (2 families with 13 transmissions). Most families have multiple transmissions from a carrier mother. Figure 1 illustrates some of the pedigrees for 
which mutant loads in hair follicles, buccal cells, or muscle have been measured. The mutant loads are the mean values of multiple measurements taken from the same tissue of an individual. It is evident that the intergenerational transmission of A3243G results in increase of mutant mtDNA in blood, hair follicles, and perhaps also in buccal cells (Fig. 1A-D). Although the carrier mother's muscle is unavailable, the mutant load in muscle of the affected is consistently higher than that in blood. ${ }^{23}$ T8993G also shows substantial intergenerational increase in mutant mtDNA (Fig. 1E). However, the T8993G pedigrees are usually small due to the rapid increase in mutant mtDNA and the severity of the mutation (Fig. 1E). Intergenerational change in T8993C mutant loads in blood and hair follicles is very small (Fig. 1F). The intergenerational transmission of A8344G shows both an increase and a decrease in mutant loads (Fig. 2). The mutant loads in the blood of three individuals (III-6, IV-11, and IV-12) were measured at two different times, 2 years apart. The mutant mtDNA increased in two of the patients, and the other one did not have significant change. This finding was different from what was observed for the A3243G mutant, which showed a longitudinal decrease of approximately $1 \%$ per year in blood. ${ }^{8}$

The results of intergenerational change of mutant mtDNA in blood for each mutation are shown in Figure 3. The transmissions to male and female offspring are illustrated separately and combined. The frequencies of decrease $(<-5 \%)$, no change ( $\geq-5$ to $\leq+5 \%)$, or increase $(>+5 \%)$ in mutant mtDNA during intergenerational transmission are depicted. If the mutant mtDNA is randomly transmitted, the distribution

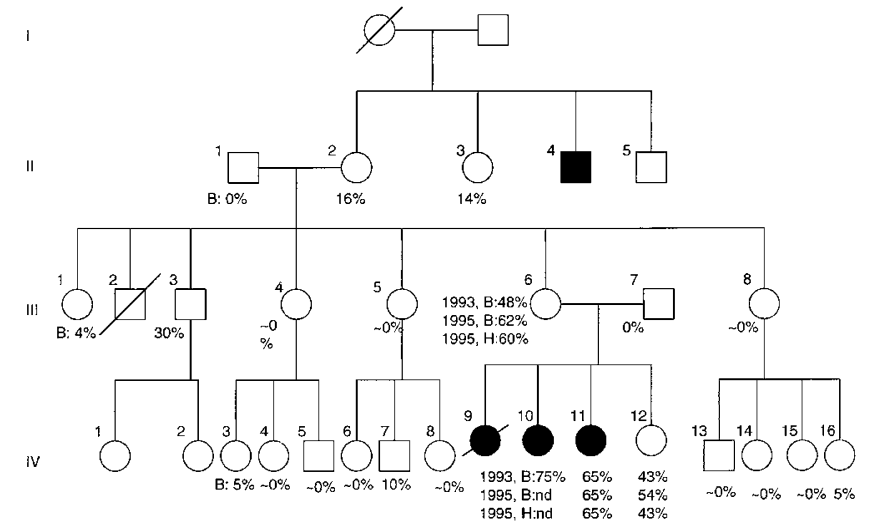

Fig. 2 Pedigree of the family with A8344G mutation showing increase and decrease in mutant loads during intergenerational transmission. The mutant loads in blood and hair follicle were measured in 1993 and 1995 in three individuals (III-6, IV-11, and IV-12) showing a longitudinal increase in mutant loads.

should be symmetrical in both the increase and the decrease sides with peak at the no change range ( $\geq-5$ to $\leq+5 \%)$. For A3243G, G8363A, T8993C, and T8993G, the distribution is asymmetrical and is in favor of transmitting the mutant mtDNA. For A8344G, the distribution appears to be symmetrical. Statistical analysis reveals that the intergenerational increase of mutant A3243G and T8993G is significant (Table 1). Although the transmissions of G8363A, A8344G, and T8993C appear to be in favor of the mutant DNA, they are not statistically significant. The transmissions of heteroplasmic mutant
A

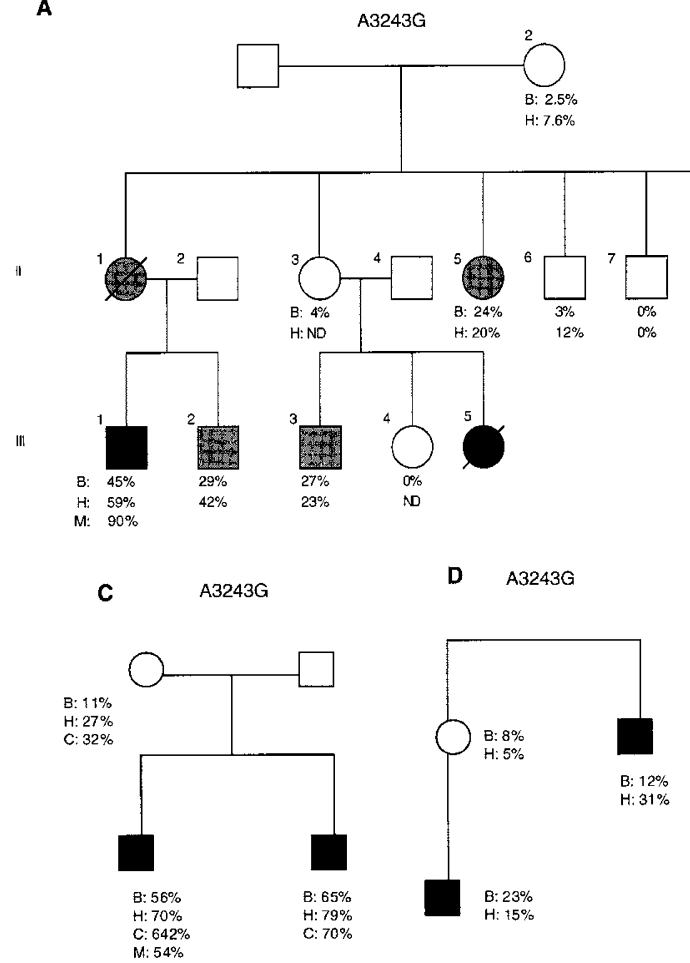

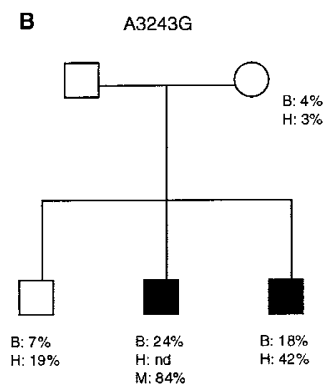

E

T8993G

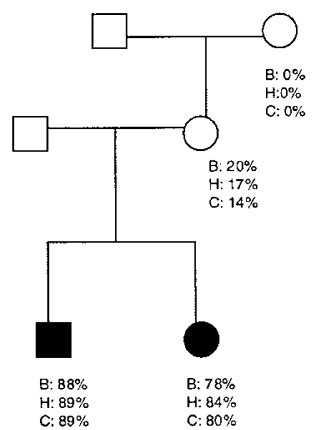

F ${ }^{\mathrm{T}} 8993 \mathrm{C}$

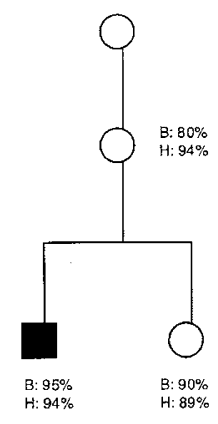

Fig. 1 Pedigrees with family members showing mutant loads in blood (B), hair follicles (H), cheek cells (C), and muscle (M). 

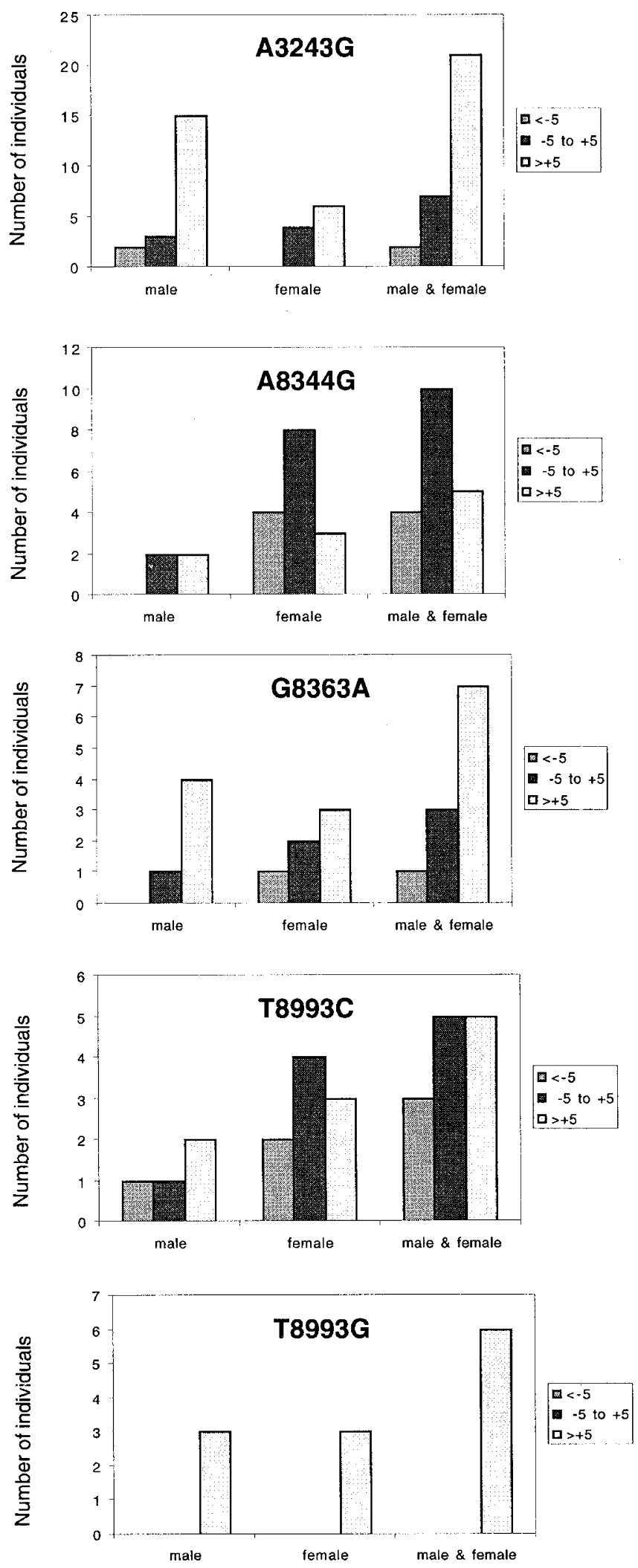

Fig. 3 Distribution of intergenerational changes during transmission of heteroplasmic mtDNA. The intergenerational changes in mutant mtDNA were categorized into three groups: decrease $(<-5 \%)$; no change $(-5$ to $+5 \%)$; and increase $(>+5 \%)$, as indicated at the right-hand side of the graphs.
Table 1

Intergenerational changes during transmission of heteroplasmic mtDNA mutations

\begin{tabular}{lccccc}
\hline & \multicolumn{4}{c}{ Transmissions to male and female offspring } \\
\cline { 2 - 6 } Mutation & $\begin{array}{c}\text { No. of } \\
\text { families }\end{array}$ & $n$ & $\begin{array}{c}\text { Mean of } \\
\text { change } \\
(\text { SD })\end{array}$ & $\begin{array}{c}\text { Wilcoxon rank sum test: } \\
\text { Corrected Z } \\
(P \text { value })\end{array}$ \\
\hline A3243G & 10 & 30 & $13.77(15.61)$ & $3.80(0.0001)$ significant \\
A8344G & 1 & 19 & $2.26(13.1)$ & $0.938(0.348)$ not significant \\
G8363A & 1 & 11 & $6.64(10.05)$ & $1.825(0.068)$ not significant \\
T8993G & 4 & 6 & $56.83(19.86)$ & $2.1(0.031)$ significant \\
T8993C & 2 & 13 & $6.23(22.17)$ & $0.687(0.492)$ not significant \\
\hline & & & &
\end{tabular}

mtDNA to male and female offspring were analyzed and compared. The results demonstrated that all five mutant mtDNA increased more in male than in female offspring. However, the difference is not statistically significant (data not shown). Because families with clinically affected individuals are more likely to be investigated, there is always a concern of an ascertainment bias. One way of resolving this problem is to omit the probands from the analysis. When this is done, the intergenerational increase of A3243G and T8993G mutant mtDNA remains statistically significant.

\section{DISCUSSION}

Our results are consistent with the observation of Chinnery et al. in that there is a significant intergenerational increase of A3243G and T8993G mutant mtDNA. The mutant mtDNA was preferentially transmitted to offspring in every family with A3243G and T8993G studied, including one large A3243G family with 10 transmissions (Fig. 1A). The number of transmissions of T8993G studied was very small, but all of them showed a substantial intergenerational increase of mutant mtDNA. The small size of the T8993G pedigree is due to the extremely rapid segregation of mutant mtDNA. Within one or two generations, the mutant mtDNA can be fixed. ${ }^{6,13,18}$ Thus, the transmission of T8993G often resulted in an appreciable intergenerational increase. ${ }^{13,14,28}$ Skewed segregation of the mutant T8993G mtDNA in human oocytes has been reported. ${ }^{13}$ In that study, six of seven oocytes analyzed had a mutant load $>95 \%$, demonstrating a significant intergenerational increase from the carrier mother who had 50\% mutant T8993G in her blood.

Although the intergenerational increase of mutant T8993G is consistently significant, it is not the case with T8993C mutation. The results from studying a family with 11 transmissions show apparent intergenerational increase with no statistical significance. Similar findings had been reported. ${ }^{19}$ The number of transmissions of T8993G and T8993C studied was small. The results may be heavily influenced by ascertainment bias, although omission of the proband from the analysis did not change the significance status. It is clear that the pattern of transmission of T8993G and T8993C mutant mtDNAs is dif- 
ferent. T8993C is a milder mutation with the alteration of leucine to proline, than T8993G with the change of leucine to arginine. Near homoplasmy of mutant T8993G causes severe impairment of oxidative phosphorylation. In addition, transmission of T8993G usually was in favor of rapid increase of mutant mtDNA within one generation. ${ }^{14}$ Transmission of increased mutant T8993G from symptomatic females with high mutant loads would be substantially hindered by reproductive fitness due to the clinical severity of the disease. In the case of T8993C, a wide range (10-90\%) of the mutant load of the transmitting female has been observed, and the transmission of heteroplasmic mtDNA could go in either direction.

The transmission of A8344G in favor of a mutant genome is insignificant. Chinnery et al. found a weak negative selection against mutant A8344G during intergenerational transmission. ${ }^{19}$ This discrepancy could be due to genetic background of the transmitting woman. In our study, 19 transmissions of A8344G are from a single family of large sibship with four generations and six carrier women (Fig. 2). The large sibship reduces the bias of selecting transmissions to clinically affected offspring, providing a good opportunity to observe random segregation. If only the transmissions from II-2 were considered, four resulted in the reduction of mutant mtDNA and two resulted in the intergeneration increase. In contrast, the carrier woman, III-6, transmitted higher mutant loads to three of her daughters, with only one resulting in a slight decrease of mutant mtDNA (Fig. 2). In addition, the longitudinal increase of mutant mtDNA in blood may also explain the observed intergenerational decrease in some cases. ${ }^{19}$

Study of a family with 11 transmissions of the G8363A mutation showed insignificant intergenerational increase of mutant mtDNA. Analysis of a second family in the report by Santorelli et al. ${ }^{4}$ showed opposite results in that all five transmissions were from the same woman to female offspring with a significant intergenerational decrease of the mutant mtDNA (mean, -3.3 ; SD, 13.8; $P=0.03$ ). Because the carrier woman already had a very high mutant load (94\%) in her blood, a further increase would result in a near-homoplasmic mutant. An oocyte with almost $100 \%$ pathogenic mutant mtDNA may not be viable. Similarly, an embryo with a high mutant load may not be able to successfully develop during early embryogenesis. Thus, the offspring with decreased mutant load is more likely to survive. Random genetic drift without selection may explain the transmission of G8363A.29 The observed intergenerational increase or decrease in mutant G8363A depends on the mutant load and genetic background of the carrier woman, similar to that in the transmission of A8344G. Studies have shown different genetic backgrounds confer a marked advantage to either mutant or wild-type mitochondrial genomes. ${ }^{30}$ The sampling effect can be minimized if a large number of transmissions are studied in a large number of families. The distribution will be more symmetrical, with an average intergenerational change around zero.

Measurement of the level of mutant mtDNA in individual primary oocytes could be a means to determine the intergenerational change. Study of the mutant loads in 82 primary oo- cytes from a woman who carried the A3243G mutation showed that the number of oocytes with mutant loads greater than the mean was approximately equal to the number with mutant loads less than the mean. ${ }^{29}$ These findings indicate that random genetic drift is the mechanism that determines the mutant load in individual oocyte..$^{29}$ If this is the case, why is the intergenerational increase in mutant A3243G consistently observed in blood leukocytes? First, there is evidence that A3243G mutant mtDNA level decreases in leukocytes, but not in skeletal muscle, upon aging. ${ }^{7}$ Longitudinal analysis revealed that the mutant load decreases at a mean overall rate of approximately $1 \%$ per year. ${ }^{8}$ Because mothers are generally at least 15 years older than their offspring, the intergenerational increase of A3243G mutant mtDNA in Table 1 may simply reflect the age difference between mothers and offspring. Second, mutant mtDNA accumulates over time. ${ }^{17,31}$ The mutant loads in muscle are usually higher than those in blood within the same individual (Ref. 23 and Fig. 1). The accumulation of mutant mtDNA may be tissue specific. ${ }^{9}$ If, in general, the mutant load in oocytes is higher than that in leukocytes, ${ }^{29}$ then the embryo will have higher mutant load to begin with. Third, the defective mitochondria may proliferate if the mutant load reaches the threshold and the proliferation is tissue specific. Thus, differences in tissue-specific segregation and genetic threshold may contribute to the pattern of intergenerational transmission. It should be noted that the rate of segregation of T8993G differs markedly from that of A3243G as mentioned above. ${ }^{13}$

Due to the severity of the mutation and the low threshold, affected individuals with high mutant load (>90\%) of A3243G in blood are rarely observed. The carrier women may be asymptomatic or mildly affected with a low $(<20 \%)$ mutant load in blood. On the contrary, it requires a much higher mutant load for A8344G, G8363A, T8993G, and T8993C to cause clinical symptoms. The asymptomatic transmitting woman could theoretically carry a higher mutant load, for example $>75 \%$. In the case of G8363A discussed above, if the mutant load is below threshold, for example, $76 \%$ in the carrier of the family we studied, the intergenerational change can go in either direction. If the mutant load reaches the threshold, for example, $94 \%$ in the second family reported by Santorelli et al., ${ }^{4}$ cells containing a lower mutant load will be preferentially selected during transmission, because any cells with a further increase in mutant load may not be able to survive. The mean intergenerational change in the second family showed a significant decrease during transmission. When the data of these two families were combined and analyzed, the means became -1 with a standard deviation of 12.6 and $P$ value of 0.84 , suggesting an insignificant intergenerational change. Thus, the transmission of G8363A is also by random genetic drift.

In conclusion, the intergenerational transmission of A3243G and T8993G results in an increase of mutant mtDNA. The intergenerational transmission of A8344G, G8363A, and T8993C depends on the mutant loads of the transmitting females. If the mutant load of the carrier is much below the threshold, the transmission of mutant mtDNA is based on random segregation. If the mutant load is above the threshold, the 
transmission may favor the selection of normal mtDNA. Due to the mild nature of the mutation, the transmission of T8993C is not particularly selected. We believe the transmission of heteroplasmic mtDNA depends on tissue-specific selection, threshold, and the molecular and biochemical consequence of the mutations. Our recent studies of mtDNA deletion syndromes suggest that there might be tissue-specific elimination of mutant mtDNA with the common 5-kb deletion but not the mtDNA with novel deletions. ${ }^{32}$ It is conceivable that similar mechanisms of tissue-specific selection of mtDNA point mutations may also take place.

\section{Acknowledgments}

This study was partially supported by a grant from the Muscular Dystrophy Association. The authors thank all the physicians who referred patients contributing to this study and all the fellows and technicians who participated in the molecular diagnosis and discussion of individual cases.

\section{References}

1. Shoffner J, Wallace D. Oxidative phosphorylation diseases. In: Scriver CR, Beaudet AL, Sly WS, Valle D, editors. The metabolic and molecular bases of inherited disease,7th ed. New York: McGraw-Hill, 1995:1535-1629.

2. DiMauro S, Bonilla E, Davidson M, Hirano M, Schon EA. Mitochondrial in neuromuscular disorders. Biochim Biophys Acta 1998;1366:199-210.

3. Chinnery PF, Turnbull DM. Mitochondrial DNA and disease. Lancet 1999; 354(suppl I):17-21.

4. Santorelli FM, Mak S-C, El-Schahawi M, Casali C, Shanske S, Baram TZ, Madrid RE, DiMauro S. Maternally inherited cardiomyopathy and hearing loss associated with a novel mutation in the mitochondrial tRNA ${ }^{\text {lys }}$ gene (G8363A). Am J Hum Genet 1996;58:933-939.

5. Chinnery PF, Howell N, Lightowlers RN, Turnbull DM. Molecular pathology of MELAS and MERRF: the relationship between mutation load and clinical phenotype. Brain 1997;120:1713-1721.

6. White SL, Collins VR, Wolfe R, Cleary MA, Shanske S, DiMauro S, Dahl HM, Thorburn DR. Genetic counseling and prenatal diagnosis for the mitochondrial DNA mutations at nucleotide 8993. Am J Hum Genet 1999;65:474-482.

7. Hart LM, Jansen JJ, Lemkes HHPJ, de Knijff P, Maassen JA. Heteroplasmy levels of a mitochondrial gene mutation associated with diabetes mellitus decrease in leukocyte DNA upon ageing. Hum Mutat 1996;7:193-197.

8. Howell N, Ghosh SS, Fahy E, Bindoff LA. Longitudinal analysis of the segregation of mtDNA mutations in heteroplasmic individuals. J Neurol Sci 2000;172:1-6.

9. Weber K, Wilson JN, Taylor L, Brierley E, Johnson MA, Turnbull DM, Bindoff LA. A new mtDNA mutation showing accumulation with time and restriction to skeletal muscle. Am J Hum Genet 1997;60:373-380.

10. Yoneda M, Chomyn A, Martinuzzi A, Hurko O, Attardi G. Marked replicative advantage of human mtDNA carrying a point mutation that causes the MELAS encephalomyopathy. Proc Natl Acad Sci U S A 1992;89:11164-11168.

11. Chinnery PF, Turnbull DM. The clinical features, investigation and management of patients with mitochondrial DNA defects. J Neurol Neurosurg Psychiatry 1997;63: $559-563$.
12. Lightowlers RN, Chinnery PF, Howell N, Turnbull DM. Mammalian mitochondrial genetics: heredity, heteroplasmy and disease. Trends Genet 1997;13:4450-4455.

13. Blok RB, Gook DA, Thorburn DR, Hahl HM. Skewed segregation of the mtDNA nt 8993 (T->G) mutation in human oocytes. Am J Hum Genet 1997;60:1495-1501.

14. Tulinius MH, Houshmand M, Larsson NG, Holme E, Oldfors A, Holmberg E, Wahlstrom J. De novo mutation in the mitochondrial ATP synthase subunit 6 gene (T8993G) with rapid segregation resulting in Leigh syndrome in the offspring. Hum Genet 1995;96:290-294.

15. Chinnery PF, Nowell N, Lightowlers RN, Turnbull DM. Genetic counseling and prenatal diagnosis for mtDNA disease. Am J Hum Genet 1998;63:1908-1910.

16. Chinnery PF, Howell N, Lightowlers RN, Turnbull DM. MELAS and MERRF: the relationship between maternal mutation load and the frequency of clinically affected offspring. Brain 1998;121:1889-1894.

17. Sue CM, Quigley A, Katsabanis S, Kapsa R, Crimmins DS, Byrne E, Morris JGL. Detection of MELAS A3243G point mutation in muscle, blood and hair follicles. J Neurol Sci 1998;161:36-39.

18. White SL, Shanske S, McGill JJ, Mountain H, Geraghty MT, DiMauro S, Dahl HH, Thorburn DR. Mitochondrial DNA mutations at nucleotide 8993 show a lack of tissue- or age-related variation. J Inherit Metab Dis 1999;22:899-914:

19. Chinnery PF, Thorburn DR, Samuels DC, White SL, Dahl HM, Turnbull DM, Lightowlers RN, Howell N. The inheritance of mitochondrial DNA heteroplasmy: random drift, selection or both? Trends Genet 2000;16:500-505.

20. Wong LJC, Senadheera D. Direct detection of multiple point mutations in mitochondrial DNA. Clin Chem 1997;43:1857-1861.

21. Liang M, Wong LJC. Yield of mtDNA mutation analysis in 2000 patients. Am J Med Genet 1998;77:395-400.

22. Isotani H, Fukumoto Y, Kawamura H, Furukawa K, Ohsawa N, Goto Y, Nishino I, Nonaka I. Hypoparathyroidism and insulin-dependent diabetes mellitus in a patient with Kearns-Sayre syndrome harbouring a mitochondrial DNA deletion. Clin Endocrinol 1996;45:637-641.

23. Wong LJC, Lam C. Alternative, noninvasive tissues for quantitative screening of mutant mitochondrial DNA. Clin Chem 1997;43:1241-1243.

24. Tatuch Y, Christodoulou J, Feigenbaum A, Clarke JTR, Wherret J, Smith C, Rudd N, Petrova-Benedict R, Robinson BH. Heteroplasmic mtDNA mutation (T- $>$ G) at 8993 can cause Leigh disease when the percentage of abnormal mtDNA is high. Am J Hum Genet 1992;50:852-858.

25. Santorelli FM, Shanske S, Jain KD, Tick D, Schon EA, DiMauro S. A T->C mutation at nt8993 of mitochondrial DNA in a child with Leigh syndrome. Neurology 1994; 44:972-974.

26. Tanno Y, Yoneda M, Tanaka K, Tanaka H, Yamazki M, Nishizawa M, Wakabayashi K, Ohama E, Tsuji S. Quantitation of heteroplasmy of mitochondrial tRNA ${ }^{\text {Leu(UUR) }}$ gene using PCR-SSCP. Muscle Nerve 1995;18:1390-1397.

27. Woolson RF. Statistical methods for the analysis of biomedical data. New York: Wiley \& Sons, 1987.

28. Harding AE, Holt IJ, Sweeney MG, Brockington M, Davis MB. Prenatal diagnosis of mitochondrial DNA ${ }^{8993 \mathrm{~T}->\mathrm{G}}$ disease. Am J Hum Genet 1992;50:629-633.

29. Brown DT, Samuels DC, Michael EM, Turnbull DM, Chinnery PF. Random genetic drift determines the level of mutant mtDNA in human primary oocytes. Am J Hum Genet 2001;68:533-536.

30. Dunbar DR, Moonie PA, Jacobs HT, Holt IJ. Different cellular backgrounds confer a marked advantage to either mutant or wild-type mitochondrial genomes. Proc Natl Acad Sci U S A 1995;92:6562-6566:

31. Michikawa Y, Mazzucchelli F, Bresolin N, Scarlato G, Attardi G. Aging-dependent large accumulation of point mutations in the human mtDNA control region for replication. Science 1999;286:774-779.

32. Wong LJC. Recognition of mitochondrial DNA deletion syndrome with non-neuromuscular multisystemic manifestation. Genet Med 2001;3:399-404. 